\title{
CÁLCULO AB INITIO DE INTENSIDADES RAMAN DINÂMICAS UTILIZANDO A TEORIA DA RESPOSTA LINEAR
}

Luciano N. Vidal e Pedro A. M. Vazquez*

Instituto de Química, Universidade Estadual de Campinas, CP 6154, 13084-971 Campinas - SP

Recebido 13/8/02; aceito 18/10/02

\begin{abstract}
AB INITIO CALCULATION OF DYNAMIC RAMAN INTENSITIES USING THE LINEAR RESPONSE THEORY. In this paper a methodology for the computation of Raman scattering cross-sections and depolarization ratios within the Placzek Polarizability Theory is described. The polarizability gradients are derived from the values of the dynamic polarizabilities computed at the excitation frequencies using ab initio Linear Response Theory. A sample application of the computational program, at the HF, MP2 and CCSD levels of theory, is presented for $\mathrm{H}_{2} \mathrm{O}$ and $\mathrm{NH}_{3}$. The results show that high correlated levels of theory are needed to achieve good agreement with experimental data.
\end{abstract}

Keywords: Raman spectra; vibrational spectroscopy; ab initio Linear Response Theory.

\section{INTRODUÇÃO}

Apesar da Teoria da Polarizabilidade de Placzek datar de $1934^{1}$, o estudo teórico de intensidades e perfis de excitação Raman tornouse viável computacionalmente apenas na última década. Atualmente, implementações computacionais para o cálculo de intensidades Raman estáticas são bastante freqüentes em pacotes de química quântica, onde estas propriedades são obtidas através de métodos $a b$ initio ou através da Teoria do Funcional de Densidade. As intensidades Raman estáticas são obtidas a partir de polarizabilidades geradas pela da ação de um campo elétrico de freqüência nula (estático), e, portanto são conhecidas como polarizabilidades estáticas. Quando as polarizabilidades tiverem sua origem associada à ação de um campo elétrico de freqüência não nula passam a ser chamadas de polarizabilidades dinâmicas, e as intensidades Raman provenientes destas polarizabilidades passam a ser designadas por intensidades Raman dinâmicas.

Cálculos empregando polarizabilidades estáticas realizados por Fleisher e Pulay ${ }^{2}$ para as moléculas de benzeno e coroneno em fase gasosa, no nível de teoria B3LYP/6-31G*, obtiveram uma concordância semiquantitativa para as intensidades Raman e razões de depolarização. Halls e Schlegel ${ }^{3}$, em um estudo comparativo envolvendo doze moléculas pequenas, utilizando os métodos ab initio Hartree-Fock, MP2 e vários funcionais (S-VWN, BLYP, B3LYP, MPW1-PW91), partindo de polarizabilidades estáticas, obtiveram seus melhores resultados no nível MP2. Entretanto, um fator comum a estes estudos baseados em polarizabilidades estáticas é que os mesmos não são capazes de reproduzir sequer o padrão de intensidades para uma particular freqüência de excitação, seja esta ressonante ou não, mesmo quando funções de base muito grandes como a augcc-pVTZ ${ }^{3}$ são utilizadas. Uma solução parcial para este problema surgiu com Helgaker e colaboradores ${ }^{4}$, que desenvolveram uma metodologia e implementação computacional, nos níveis de teoria Hartree-Fock e MCSCF, para o cálculo de intensidades Raman dinâmicas de moléculas em fase gasosa, a partir de polarizabilidades calculadas na mesma freqüência de excitação utilizada para obter o espectro (polarizabilidades dinâmicas). As polarizabilidades dinâmi-

*e-mail: vazquez@iqm.unicamp.br cas são obtidas através da Teoria da Resposta Linear (LRT) ${ }^{5-7}$ e o gradiente da polarizabilidade, utilizado no cálculo das intensidades Raman dinâmicas dentro da teoria de Placzek, é obtido numericamente. Recentemente, Champagne ${ }^{8,9}$ introduziu a primeira implementação computacional de derivadas geométricas analíticas das polarizabilidades, para o nível Hartree-Fock, que permitem reduzir substancialmente o tempo computacional necessário para o cálculo das intensidades Raman dinâmicas. Caillie et al. ${ }^{10}$ apresenta o cálculo de intensidades Raman para um conjunto de dez moléculas pequenas utilizando métodos $a b$ initio Hartree-Fock e MP2 e vários funcionais (B3LYP, PBE0, PBE e B97-1), com funções de base de Sadlej ${ }^{11,12}$ e gradientes de polarizabilidades estáticas (todos os métodos) e dinâmicas (somente DFT e HF). Neste trabalho, diferenças de até $15 \%$ entre cálculos com polarizabilidades estáticas e dinâmicas foram encontradas. Apesar disto, é difícil avaliar cada método frente a resultados experimentais, devido às incertezas que acompanham estas medidas. Conclui-se deste estudo que a correlação eletrônica é extremamente importante para o cálculo desta propriedade e que métodos mais sofisticados são necessários para obter-se uma concordância quantitativa com dados experimentais. Partindo de gradientes numéricos e utilizando a Teoria da Resposta no cálculo de polarizabilidades dinâmicas, Tomasi ${ }^{13-15}$, em trabalhos recentes, divulgou um método baseado no modelo PCM para simular o espectro Raman de sistemas sob efeito de solvente. Estudos realizados de intensidades Raman de moléculas diatômicas utilizando as metodologias correlacionadas SOPPA ${ }^{16}$ e TDGI $^{17}$ apresentam resultados de qualidade comparável e ambos justificam o uso de métodos mais sofisticados para o cálculo desta propriedade. Surpreendentemente, resultados obtidos a partir do método não correlacionado $\mathrm{CHF}^{17}$ fornecem melhores intensidades quando comparadas aos resultados obtidos com o método SOPPA ${ }^{17}$. Para níveis mais elevados de teoria, Pecul et al. ${ }^{18,19}$ realizaram um estudo de convergência de funções de base (aug-cc-pVnZ e d-aug-cc-pVnZ, $\mathbf{n}=2,3,4,5$ ) em várias hierarquias do nível de teoria Coupled Cluster (CCS,CC2,CCSD e CC3) sobre a polarizabilidade, seu gradiente e seções diferenciais de choque de algumas moléculas diatômicas, mostrando que o gradiente da polarizabilidade e a seção diferencial de choque são muito mais sensíveis ao tamanho das funções de base e à correlação eletrônica em comparação com a polarizabilidade. No entanto, estas funções de base são muito grandes para serem utilizadas em cálculos 
de intensidades Raman dinâmicas para a maioria dos sistemas de interesse químico e espectroscópico. Abordando especificamente a convergência de funções de base, Helgaker et al. ${ }^{4}$ determinou que no nível Hartree-Fock de teoria seria necessário o uso do conjunto de bases aug-cc-pVDZ para alcançar convergência numérica das intensidades absolutas e do conjunto aug-cc-pVTZ para, simultaneamente, obter convergência numérica das freqüências vibracionais; de Miranda $^{20}$, por outro lado, analisando as intensidades relativas dos perfis de excitação do ânion $\mathrm{C}_{4} \mathrm{O}_{4}^{-2}$, observou que estas convergem mais rapidamente neste caso, do que as intensidades absolutas e sem deixar de reproduzir corretamente os padrões de intensificação de cada modo normal observados experimentalmente. Em geral, estudos ab initio e com a Teoria do Funcional da Densidade sobre a influência do conjunto de bases na qualidade das intensidades Raman estáticas, polarizabilidades estáticas e dinâmicas ${ }^{3,21,22}$ têm concluído que as bases de Sadlej ${ }^{11,12}$ representam o melhor compromisso entre a qualidade dos resultados e o custo computacional envolvido.

O estudo teórico de intensidades e perfis de excitação Raman empregando métodos ab initio e a Teoria da Resposta é promissor, no entanto, os avanços nos métodos teóricos de cálculos das polarizabilidades moleculares dinâmicas têm sido mais rápidos que a implementação computacional necessária para utilizar estas polarizabilidades no cálculo de intensidades Raman no mesmo nível de teoria. Portanto, este trabalho tem como objetivo apresentar uma metodologia para o cálculo de intensidades Raman em níveis arbitrários de teoria, bem como resultados obtidos a partir da implementação computacional desta. Este artigo é organizado da seguinte maneira: na próxima seção são apresentados os principais resultados da teoria de polarizabilidade de Placzek para intensidades Raman em fase gasosa. A seguir são apresentados os métodos intermediários necessários ao cálculo destas intensidades e, por último, o programa é empregado no cálculo de intensidades Raman dinâmicas das moléculas de $\mathrm{H}_{2} \mathrm{O}$ e $\mathrm{NH}_{3}$, verificando efeitos de correlação eletrônica e funções de base.

\section{TEORIA}

\section{Seções diferenciais de choque Raman e razões de depolarização}

O espectro Raman é comumente expresso em termos de seções diferenciais de choque ( $d \sigma / d \Omega$ ), onde $\Omega$ representa o ângulo sólido que é atravessado por um feixe cônico de radiação espalhada. Segundo a teoria da polarizabilidade de Placzek ${ }^{23}$, a seção diferencial de choque Raman Stokes correspondente ao $k$-ésimo modo normal de vibração, medida perpendicularmente à direção de propagação do feixe de luz incidente $(\pi / 2)$, para um conjunto de moléculas em fase gasosa, é dada por $^{24}$ :

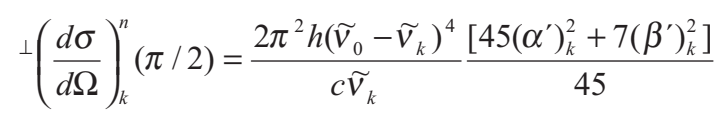

$\mathrm{O}$ índice $\perp$ indica que a radiação incidente é linearmente polarizada e sua direção de polarização é perpendicular ao plano espalhador. O plano espalhador é o plano que contém a direção de propagação da radiação incidente e a direção de observação. $\mathrm{O}$ índice $n$ informa que a radiação espalhada é medida sem o uso de polarizadores. $\widetilde{v}_{0}$ e $\widetilde{v}_{k}$ correspondem, respectivamente, aos números de onda das frequiências de excitação e do $k$-ésimo modo normal de vibração, $h$ é a constante de Planck e $c$ a velocidade da luz. A razão de depolarização para a radiação incidente polarizada perpendicularmente ao plano espalhador, $\rho \perp(\pi / 2)$, é definida como: $\rho_{\perp}(\pi / 2)=\frac{\perp\left(\frac{d \sigma}{d \Omega}\right)_{k}^{\|}(\pi / 2)}{\perp\left(\frac{d \sigma}{d \Omega}\right)_{k}^{\perp}(\pi / 2)}=\frac{3\left(\beta^{\prime}\right)_{k}^{2}}{45\left(\alpha^{\prime}\right)_{k}^{2}+4\left(\beta^{\prime}\right)_{k}^{2}}$

e, portanto, mede as mudanças ocorridas na polarização da radiação do feixe espalhado com relação ao feixe incidente. Os gradientes da polarizabilidade média, $\alpha^{\prime}$, e da anisotropia, $\beta^{\prime}$, referentes aos modos normais de vibração, são obtidos a partir das expressões:

$$
\begin{aligned}
\left(\alpha^{\prime}\right)_{k}= & \frac{1}{3}\left(\alpha_{x x, k}^{\prime}+\alpha_{y y, k}^{\prime}+\alpha_{z z, k}^{\prime}\right) \\
\left(\beta^{\prime}\right)_{k}^{2}= & \frac{1}{2}\left\{\left(\alpha_{x x, k}^{\prime}-\alpha_{y y, k}^{\prime}\right)^{2}+\left(\alpha_{y y, k}^{\prime}-\alpha_{z z, k}^{\prime}\right)^{2}+\left(\alpha_{z z, k}^{\prime}-\alpha_{x x, k}^{\prime}\right)^{2}+\right. \\
& \left.6\left[\left(\alpha_{x y, k}^{\prime}\right)^{2}+\left(\alpha_{y z, k}^{\prime}\right)^{2}+\left(\alpha_{z x, k}^{\prime}\right)_{2}\right]\right\}
\end{aligned}
$$

onde $\alpha^{\prime}{ }_{m n, k}$ representa a variação da componente $\alpha_{m n}$ do tensor polarizabilidade em relação ao modo normal $k$, sendo calculada a partir da expressão:

$\alpha_{m n, k}^{\prime}=\left(\frac{\partial \alpha_{m n}}{\partial Q_{k}}\right)_{e q}=\sum_{i=1}^{3 N}\left[\left(\frac{\partial \alpha_{m n}}{\partial \xi_{i}}\right) \cdot l_{i, k}^{W}\right]$

Nesta equação, $N$ corresponde ao número de átomos da molécula, $\xi$ representa as coordenadas cartesianas atômicas, $l_{i, k}^{W}$ corresponde a $i$-ésima componente, ponderada em massa, do autovetor do modo normal $k, \mathbf{L}_{k}$, i.e.

$\mathbf{L}_{k}^{W}=\left(\frac{l_{x_{1}, k}}{\sqrt{m_{1}}} ; \frac{l_{y_{1}, k}}{\sqrt{m_{1}}} ; \frac{l_{z_{1}, k}}{\sqrt{m_{1}}} \cdots \frac{l_{z_{N}, k}}{\sqrt{m_{N}}}\right)$

sendo $m$ a massa atômica; $\alpha_{m n}$ representa uma das componentes do tensor polarizabilidade, calculado para uma dada frequiência de excitação $\tilde{v}_{0}$. As polarizabilidades usadas nestas equações devem ser divididas por $4 \pi \varepsilon_{0}$ ( $\varepsilon_{0}$ é a permissividade do vácuo). O índice $e q$ indica que a diferenciação é feita na geometria de equilíbrio.

A diferenciação da componente $\alpha_{m n}$ com relação à coordenada $\xi_{i}$ é feita numericamente no programa, sendo a $i$-ésima componente do gradiente de $\alpha_{m n}$ dada por $^{25}$ :

$$
\left(\frac{\partial \alpha_{m n}}{\partial \xi_{i}}\right)=\frac{\alpha_{m n}^{\xi_{i}+\delta}-\alpha_{m n}^{\xi_{i}-\delta}}{2 \delta}
$$

Nesta expressão $\delta$ representa o deslocamento cartesiano.

Por fim, a seção diferencial de choque ${ }^{\perp}(d \sigma / d \Omega)_{k}^{n}(\pi / 2)$ necessita ainda da correção termodinâmica de temperatura dada pela distribuição populacional de Boltzmann. A fração de moléculas presentes no estado fundamental, $f_{\widetilde{v}_{k}}$, de cada modo normal de vibração $k$, utilizando a aproximação harmônica, é dada $\operatorname{por}^{26,27}$ :

$$
f_{\widetilde{v}_{k}}=\frac{\exp \left(-h c \widetilde{v_{k}} / k T\right)}{Q_{v i b, k}^{h a r m}}
$$

sendo $Q_{v i b, k}^{\text {harm }}$ a função de partição vibracional harmônica:

$Q_{v i b, k}^{\text {harm }}=\frac{\exp \left(-\Theta_{\mathrm{v}} / 2 T\right)}{1-\exp \left(-\Theta_{\mathrm{v}} / T\right)}$ 
onde $\Theta=h c \tilde{v}_{k} / k$. Como à temperatura de $298 K, \Theta_{v} / \mathrm{T}$ é pequeno para a maioria das frequiências vibracionais, a correção de temperatura é feita simplesmente multiplicando $(d \sigma / d \Omega)$ por $f_{\widetilde{v}_{k}}$.

\section{Modos normais de vibração}

Segundo exposto na seção anterior, as coordenadas normais e respectivas frequiências vibracionais são necessárias ao cômputo de seções de choque e razões de depolarização. Desta forma, o cálculo de modos normais de vibração ${ }^{28}$ foi também implementado, possibilitando o uso de autovetores e autovalores dos modos normais de vibração no mesmo nível de teoria empregado no cálculo das polarizabilidades.

A matriz hessiana é obtida numericamente, sendo cada elemento $f_{i j}=\partial^{2} E / \partial \xi_{i} \partial \xi_{j}$ aproximado por $^{25}$ :

$f_{i j}=\frac{\left(E_{\xi_{j}+\delta}^{\xi_{i}+\delta}-E_{\xi_{j}+\delta}^{\xi_{i}-\delta}\right)+\left(E_{\xi_{j}-\delta}^{\xi_{i}-\delta}-E_{\xi_{j}-\delta}^{\xi_{i}+\delta}\right)}{4 \delta^{2}}$

Nesta equação, $E$ corresponde à energia total, $\xi_{i}$ representa uma coordenada cartesiana atômica e $\delta$ o deslocamento cartesiano, cujo "default" é $\delta=10^{-2}$ bohr (O uso de coordenadas cartesianas na construção da matriz hessiana e também do gradiente da polarizabilidade ao invés de coordenadas normais é vantajoso porque evita inconvenientes como: (1) $Q_{k}$ e $\alpha^{\prime}{ }_{m n, k}$ serem dependentes de substituições isotópicas; (2) as coordenadas normais mudam de uma para outra molécula, o que dificulta a comparação das quantidades $Q_{k}$ e $\alpha^{\prime}{ }_{m n, k}$. Esta matriz, $\mathbf{F}_{\xi}$, é então ponderada em massa,

$\mathbf{F}_{\mathbf{W}}=\mathbf{M}^{-1 / 2} \mathbf{F}_{\xi} \mathbf{M}^{-1 / 2}$

onde

$\mathbf{M}^{-1 / 2}=\left(\begin{array}{cccccc}m_{1}^{-1 / 2} & & & & & 0 \\ & m_{1}^{-1 / 2} & & & & \\ & & m_{1}^{-1 / 2} & & & \\ & & & m_{2}^{-1 / 2} & \ddots & \\ 0 & & & & & m_{N}^{-1 / 2}\end{array}\right)$

sendo $m$ a massa atômica, gerando a matriz $\mathbf{F}_{\mathbf{w}}$ que é então diagonalizada, obtendo-se seus autovalores $\lambda_{k}$ e autovetores $\mathbf{L}_{\mathbf{k}}$ a partir da equação secular:

$\left|\mathbf{F}_{\mathrm{w}}-\lambda \mathbf{E}\right|=\mathbf{0}$

O número de onda de cada modo normal de vibração é calculado a partir da equação:

$\widetilde{v}_{k}=\sqrt{\frac{\lambda_{k}}{4 \pi^{2} c^{2}}}$

\section{Polarizabilidades dinâmicas a partir da Teoria da Resposta}

A interação de um sistema quântico com um campo dependente do tempo pode ser tratada a partir das Funções de Resposta ${ }^{6}$. As polarizabilidades dinâmicas utilizadas no cálculo do gradiente da polarizabilidade são obtidas a partir da Função de Resposta Linear. A $\alpha_{m, n}$-ésima componente do tensor polarizabilidade dependente da frequiência é obtida da parte real da função de resposta linear ${ }^{6}$ :

$\alpha_{m, n}\left(\omega_{0}\right)=\left\langle\left\langle\mu^{m} ; \mu^{n}\right\rangle\right\rangle_{\omega_{0}}=\sum_{j>0}\left(\frac{\left\langle 0\left|\mu^{m}\right| j\right\rangle\left\langle j\left|\mu^{n}\right| 0\right\rangle}{\omega_{0}-\omega_{j}}-\frac{\left\langle 0\left|\mu^{n}\right| j\right\rangle\left\langle j\left|\mu^{m}\right| 0\right\rangle}{\omega_{0}+\omega_{j}}\right)$ onde $\omega_{0}$ é a frequiência de excitação do campo elétrico periódico incidente e $\omega_{j}=E_{0}-E_{j}$ denota a diferença de energia entre o autoestado exato $|0\rangle$ de $H_{0}$ em $t=-\infty: H_{0}|0\rangle=E_{0}|0\rangle$, e os $j$-ésimos autoestados exatos residuais: $H_{0}|j\rangle=E_{J}|j\rangle$.

\section{DETALHES COMPUTACIONAIS}

Os cálculos de seções diferenciais de choque Raman e razões de depolarização foram realizados com o programa desenvolvido, PLACZEK, seguindo a metodologia já apresentada. As polarizabilidades foram calculadas com o programa de estrutura eletrônica DALTON $^{29}$ versão 1.2.1, que apresenta uma implementação da função de resposta linear para diversos níveis de teoria. O gradiente cartesiano numérico das polarizabilidades foi obtido utilizando um deslocamento $\delta=10^{-4} \mathrm{bohr}$. Os autovetores e autovalores dos modos normais de vibração foram calculados com o programa PLACZEK, sendo que as energias necessárias à determinação da matriz de constantes de força foram obtidas com o programa DALTON. O tamanho do deslocamento cartesiano empregado nestes cálculos foi $\delta=$ $10^{-2}$ bohr. As moléculas utilizadas neste estudo foram $\mathrm{H}_{2} \mathrm{O}$ e $\mathrm{NH}_{3}$, excitadas em $514 \mathrm{~nm}$. Empregou-se os níveis de teoria HF, MP2 e CCSD, com funções de base de Sadlej e aug-cc-pVTZ.

\section{RESULTADOS E DISCUSSÃO}

\section{Dependência dos modos normais de vibração e gradientes da polarizabilidade com o nível de teoria}

A metodologia apresentada para determinação das intensidades Raman, que são obtidas a partir da variação da polarizabilidade com relação às coordenadas normais, $\left(\partial \alpha / \partial Q_{k}\right)$ e das frequiências dos modos normais de vibração, é composta por duas etapas computacionalmente independentes: (1) determinação das coordenadas normais e frequiências vibracionais e (2) cálculo de gradientes cartesianos atômicos do tensor polarizabilidade. Nesta seção, o comportamento de cada uma destas etapas será investigado frente a diferentes níveis teóricos, o que servirá de base para compreensão da influência deste fator sobre as intensidades Raman apresentadas na próxima seção.

Abordando inicialmente o efeito do nível teórico sobre os modos normais de vibração, a Tabela 1 apresenta frequiências vibracionais calculadas para $\mathrm{H}_{2} \mathrm{O}$ e $\mathrm{NH}_{3}$ com os métodos $\mathrm{HF}$, MP2 e CCSD, utilizando funções de base de Sadlej. Os resultados experimentais contidos nesta tabela mostram que o método HF produz frequiências vibracionais superestimadas, tendo como conseqüência a atenuação das bandas Raman. Naturalmente as intensidades resultantes irão também depender dos gradientes $\left(\partial \alpha / \partial Q_{k}\right)$ do tensor polarizabilidade, sendo possível que esta atenuação não seja observada. Com relação às

Tabela 1. Freqüências vibracionais $\left(\mathrm{em}^{-1}\right)$ calculadas e experimentais

\begin{tabular}{lcccc}
\hline $\mathrm{H}_{2} \mathrm{O}$ & $\tilde{v}_{1}\left(A_{1}\right)$ & $\tilde{v}_{2}\left(A_{1}\right)$ & $\tilde{v}_{3}\left(B_{2}\right)$ & \\
\hline HF/Sadlej & 1760 & 4128 & 4242 & \\
MP2/Sadlej & 1644 & 3800 & 3938 & \\
CCSD/Sadlej & 1669 & 3815 & 3934 & \\
Experimental $^{31}$ & 1595 & 3657 & 3756 & \\
\hline $\mathrm{NH}_{3}$ & $\tilde{v}_{1}\left(A_{1}\right)$ & $\tilde{v}_{2}(E)$ & $\tilde{v}_{3}\left(A_{1}\right)$ & $\tilde{v}_{4}(E)$ \\
\hline HF/Sadlej $_{\text {MP2/Sadlej }}^{1129}$ & 1784 & 3693 & 3826 \\
CCSD/Sadlej $_{\text {Experimental }}{ }^{31}$ & 1077 & 1663 & 3475 & 3631 \\
\hline
\end{tabular}


frequiências obtidas com os métodos MP2 e CCSD, observa-se uma convergência de métodos, com ambos apresentando um bom desempenho. As principais diferenças entre as freqüências calculadas MP2 e CCSD e os dados experimentais surgem nos modos de estiramento simétrico e assimétrico, devido a efeitos de anarmonicidade. Portanto, o método MP2 torna-se uma boa alternativa para o cálculo de frequiências vibracionais, já que a qualidade dos resultados CCSD não justifica sua maior demanda computacional. Além disso, o método HF com freqüências vibracionais escalonadas pode também representar uma boa opção para o cálculo desta propriedade.

Os gradientes da polarizabilidade média $\left(\alpha^{\prime}\right)$ e da anisotropia $\left(\beta^{\prime}\right)$, com relação a cada coordenada normal de vibração $\left(Q_{k}\right)$, para $\mathrm{H}_{2} \mathrm{O}$ e $\mathrm{NH}_{3}$, obtidos nos níveis HF, MP2 e CCSD, encontram-se na Tabela 2. Para ambas moléculas, observa-se um maior gradiente para os modos de estiramento, indicando que o espectro Raman será dominado por estas bandas. Observa-se que o método HF é responsável pelos menores gradientes o que, somado às freqüências vibracionais superestimadas, promoverá uma atenuação das intensidades Raman com relação aos métodos correlacionados. Ao contrário do comportamento observado para os modos normais de vibração, não se observa a convergência dos gradientes do tensor polarizabilidade na seqüência $\mathrm{HF} \rightarrow \mathrm{MP} 2 \rightarrow \mathrm{CCSD}$, ao contrário, o método MP2 parece superestimar estes gradientes, enquanto que o HF os subestima. Portanto, verifica-se que os gradientes da polarizabilidade são fortemente afetados pelo nível teórico.

Na próxima seção, intensidades Raman calculadas serão confrontadas com valores experimentais, possibilitando uma avaliação mais eficaz de cada método abordado nesta seção, onde pela primeira vez os métodos correlacionados MP2 e CCSD são empregados no cálculo desta propriedade a partir de gradientes não-estáticos de polarizabilidade, para sistemas com mais de dois átomos.

\section{Intensidades Raman calculadas versus experimentais}

A comparação de intensidades Raman absolutas com dados experimentais é sempre problemática devido às grandes incertezas que acompanham estas medidas. Segundo Myers ${ }^{30}$, a precisão que acompanha estas medidas não é maior que $\pm 10-15 \%$, mesmo nos melhores casos. Além disso, a conversão para valores absolutos é outra fonte adicional de erros ${ }^{18}$. A Tabela 3 contém intensidades Raman e razões de depolarização, obtidas nos níveis HF/LRT, MP2/SOPPA e CCSD/LRT, para $\mathrm{H}_{2} \mathrm{O}$ e $\mathrm{NH}_{3}$, excitadas em $514 \mathrm{~nm}$. Funções de base de Sadlej e aug-cc-pVTZ foram empregadas. Ambas são acrescidas de funções difusas. Nos cálculos utilizando a base aug-cc-pVTZ, os modos normais de vibração foram obtidos com as funções de base de Sadlej, devido à grande demanda computacional exigida no cálculo da matriz de constantes de força, sendo a base aug-cc-pVTZ utilizada exclusivamente no cômputo das polarizabilidades. Os resultados nesta tabela sugerem que ambos os métodos HF e MP2 são inadequados ao cálculo de intensidades Raman, o primeiro subestimando intensidades e o segundo superestimando. Todos os cálculos CCSD/LRT apresentaram um ótimo desempenho, com concordâncias com o experimental quase sempre quantitativas. Quanto a razões de depolarização, todos os métodos apresentaram boa exatidão.

Outro aspecto abordado foi a contribuição dos elétrons internos para as propriedades Raman estudadas. Para tanto, cálculos seguindo as metodologias CCSD/FCO/aug-cc-pVTZ (caroço congelado), CCSD/aug-cc-pVTZ e CCSD/aug-cc-pCVTZ foram realizados para ambas moléculas. Observou-se que os elétrons do caroço exercem pouca influência sobre estas propriedades, dando condições para que cálculos com similar exatidão e computacionalmente mais baratos sejam realizados.

Com relação ao conjunto de funções de base, observou-se que as

Tabela 2. Gradientes da polarizabilidade média $\left(\alpha^{\prime}\right)$ e da anisotropia $\left(\beta^{\prime}\right)$, expressos em u.a. x 100

\begin{tabular}{|c|c|c|c|c|}
\hline & $v_{1}\left(A_{1}\right)$ & $v_{2}\left(A_{1}\right)$ & $v_{3}\left(B_{2}\right)$ & \\
\hline $\mathrm{H}_{2} \mathrm{O}$ & $\alpha^{\prime} / \beta^{\prime}$ & $\alpha^{\prime} / \beta^{\prime}$ & $\alpha^{\prime} / \beta^{\prime}$ & \\
\hline HF/Sadlej & $0,000 / 0,084$ & $1,169 / 1,219$ & $0,000 / 2,419$ & \\
\hline MP2/Sadlej & $0,001 / 0,106$ & $2,268 / 1,116$ & $0,000 / 3,352$ & \\
\hline \multirow[t]{2}{*}{ CCSD/Sadlej } & $0,001 / 0,100$ & $1,766 / 1,101$ & $0,000 / 2,900$ & \\
\hline & $v_{1}\left(A_{1}\right)$ & $v_{2}(E)$ & $v_{3}\left(A_{1}\right)$ & $v_{4}(E)$ \\
\hline $\mathrm{NH}_{3}$ & $\alpha^{\prime} / \beta^{\prime}$ & $\alpha^{\prime} / \beta^{\prime}$ & $\alpha^{\prime} / \beta^{\prime}$ & $\alpha^{\prime} / \beta^{\prime}$ \\
\hline HF/Sadlej & $0,003 / 0,045$ & $0,000 / 0,106$ & $2,278 / 0,821$ & $0,000 / 4,029$ \\
\hline MP2/Sadlej & $0,029 / 0,220$ & $0,000 / 0,113$ & $3,558 / 0,383$ & $0,000 / 5,200$ \\
\hline CCSD/Sadlej & $0,022 / 0,173$ & $0,000 / 0,126$ & $3,157 / 0,437$ & $0,000 / 4,638$ \\
\hline
\end{tabular}

Tabela 3. Intensidades Raman calculadas e experimentais ${ }^{\perp} I^{n}(\pi / 2)$ (em $\AA^{4} /$ u.m.a.), razões de depolarização $\rho_{\perp}(\pi / 2)$ (entre parênteses) e frequiências vibracionais experimentais ${ }^{31}\left(\mathrm{em} \mathrm{cm}^{-1}\right)$, para as moléculas de $\mathrm{H}_{2} \mathrm{O}$ e $\mathrm{NH}_{3}$

\begin{tabular}{|c|c|c|c|c|c|c|c|c|}
\hline $\mathrm{H}_{2} \mathrm{O}$ & $\tilde{v}_{k}$ & $\begin{array}{r}\mathrm{HF} / \\
\text { Sadlej }\end{array}$ & $\begin{array}{l}\text { MP2/ } \\
\text { Sadlej }\end{array}$ & $\begin{array}{r}\text { CCSD/ } \\
\text { Sadlej }\end{array}$ & $\begin{array}{l}\mathrm{CCSD} / \mathrm{FCO} / \\
\text { aug-cc-pVTZ }\end{array}$ & $\begin{array}{r}\mathrm{CCSD} / \\
\text { aug-cc-pVTZ }\end{array}$ & $\begin{array}{r}\text { CCSD/ } \\
\text { aug-cc-pCVTZ }\end{array}$ & Expt. ${ }^{8}$ \\
\hline$v_{1}\left(A_{1}\right)$ & 1595 & $0,82(0,73)$ & $1,10(0,69)$ & $1,05(0,69)$ & $1,11(0,75)$ & $1,09(0,75)$ & $1,11(0,75)$ & $0,9 \pm 0,02(0,74)$ \\
\hline$v_{2}\left(A_{1}\right)$ & 3657 & $94,76(0,06)$ & $157,16(0,03)$ & $124,62(0,04)$ & $117,15(0,04)$ & $115,64(0,04)$ & $116,31(0,04)$ & $108 \pm 14(0,03)$ \\
\hline$v_{3}\left(B_{2}\right)$ & 3756 & $26,68(0,75)$ & $33,54(0,75)$ & $29,02(0,75)$ & $28,50(0,75)$ & $28,04(0,75)$ & $28,24(0,75)$ & $19,2 \pm 2,1(0,75)$ \\
\hline $\mathrm{NH}_{3}$ & $\tilde{v}_{k}$ & $\begin{array}{r}\mathrm{HF} / \\
\text { Sadlej }\end{array}$ & $\begin{array}{l}\text { MP2/ } \\
\text { Sadlej }\end{array}$ & $\begin{array}{r}\text { CCSD/ } \\
\text { Sadlej }\end{array}$ & $\begin{array}{l}\mathrm{CCSD} / \mathrm{FCO} / \\
\text { aug-cc-pVTZ }\end{array}$ & $\begin{array}{r}\mathrm{CCSD} / \\
\text { aug-cc-pVTZ }\end{array}$ & $\begin{array}{r}\mathrm{CCSD} / \\
\text { aug-cc-pCVTZ }\end{array}$ & Expt. $^{8}$ \\
\hline$\overline{v_{1}\left(A_{1}\right)}$ & 950 & $1,19(0,42)$ & $4,04(0,30)$ & $3,18(0,31)$ & $2,08(0,32)$ & $2,21(0,30)$ & $2,03(0,31)$ & \\
\hline$v_{2}(E)$ & 1627 & $1,97(0,75)$ & $2,27(0,75)$ & $2,51(0,75)$ & $2,28(0,75)$ & $2,23(0,75)$ & $2,26(0,75)$ & \\
\hline$v_{3}\left(A_{1}\right)$ & 3337 & $170,64(0,02)$ & $232,61(0,01)$ & $207,44(0,01)$ & $190,03(0,01)$ & $187,42(0,01)$ & $188,29(0,01)$ & $182 \pm 2$ \\
\hline$v_{4}(E)$ & 3444 & $92,24(0,75)$ & $104,07(0,75)$ & $92,80(0,75)$ & $85,26(0,75)$ & $83,21(0,75)$ & $84,10(0,75)$ & \\
\hline
\end{tabular}


de Sadlej, que exigem menor demanda computacional, mantiveram o compromisso entre a qualidade e o tempo de processamento, sendo uma alternativa viável para sistemas com maior número de elétrons.

\section{CONCLUSÕES}

Neste trabalho é apresentada uma metodologia para o cálculo de intensidades de espalhamento Raman de sistemas moleculares poliatômicos, baseada na teoria da polarizabilidade de Placzek ${ }^{23}$, utilizando polarizabilidades dinâmicas provenientes da Teoria da Resposta Linear ${ }^{6}$. Através da implementação computacional desta metodologia, estudos de efeitos de correlação eletrônica e do conjunto de funções de base revelaram a existência de uma forte dependência das intensidades com o nível de teoria, onde os métodos Hartree-Fock e MP2 mostraram-se ineficazes para este tipo de propriedade, porém, o CCSD apresentou resultados com grande exatidão, sendo um método promissor para este tipo de tarefa. Com respeito às funções de base, as de Sadlej ${ }^{11,12}$ exibiram a melhor relação exatidão versus tempo de CPU.

Este estudo para sistemas poliatômicos somado aos trabalhos de Pecul $^{18,19}$ (somente moléculas diatômicas) representam os primeiros relatos acerca do efeito da correlação eletrônica sobre gradientes do tensor polarizabilidade $\left(\partial \alpha / \partial Q_{k}\right)$, precursor das intensidades Raman dentro da Teoria de Placzek, obtidos a partir de polarizabilidades dinâmicas, em que se demonstra a necessidade de métodos correlacionados sofisticados para se obter uma concordância quantitativa com dados experimentais. Esperamos que este trabalho estimule o número de implementações computacionais desta metodologia em programas $a b$ initio, não somente pela qualidade dos resultados obtidos mas, também, porque se trata de uma área da espectroscopia onde há grandes dificuldades experimentais no que se refere a valores de intensidades, especialmente absolutos.

\section{AGRADECIMENTOS}

Os autores agradecem o apoio financeiro da FAPESP (projetos 1998/06871-1 e 02/05936-0) e da FAEP/UNICAMP (projetos 0692/ 00 e 0901/01). L. N. Vidal agradece aos professores N. H. Morgon, R. Custodio e Y. Hase pelas valiosas discussões e orientação na disciplina QF935.

\section{REFERÊNCIAS}

1. Placzek, G. Em Handbuch der Radiologie; Marx, E., ed.; Akademische Verlagsgesellschaft: Leipzig, 1934, vol. 6, p. 205.
2. Fleicher, U.; Pulay, P.; J. Raman Spectrosc. 1998, 29, 473.

3. Halls, M. D.; Schlegel, H. B.; J. Chem. Phys. 1999, 111, 8819.

4. Helgaker, T.; Ruud, K.; Bak, K. L.; Jorgensen, P.; Olsen, J.; Faraday Discuss. 1994, 99, 165.

5. Gauss, J. Em Modern Methods and Algorithms of Quantum Chemistry; Grotendorst, J., ed.; $2^{\text {th }}$ ed., John von Neumann Institute for Computing: Jülich, 2000, vol. 3.

6. Olsen, J.; Jorgensen, P.; J. Chem. Phys. 1985, 82, 3235.

7. Jorgensen, P.; Jensen, H. J. A.; J. Chem. Phys. 1988, 89, 3654.

8. Quinet, O.; Champagne, B.; J. Chem. Phys. 2001, 115, 6293.

9. Quinet, O.; Champagne, B.; Kirtman, B.; J. Comput. Chem. 2001, 22, 1920.

10. Caillie, C. V.; Amos, R. D.; Phys. Chem. Chem. Phys. 2000, 2, 2123.

11. Sadlej, A. J.; Collect. Czech. Chem. Commun. 1995, 53, 1988.

12. Sadlej, A.; Theor. Chim. Acta 1991, 79, 123.

13. Corni, S.; Tomasi, J.; Chem. Phys. Lett. 2001, 342, 135.

14. Cappelli, C.; Corni, S.; Tomasi, J.; J. Chem. Phys. 2001, 115, 5531.

15. Corni, S.; Cappelli, C.; Cammi, R.; Tomasi, J.; J. Phys. Chem. A 2001, 105, 8310 .

16. Oddershede, J.; Svendsen, E. N.; Chem. Phys. 1982, 64, 359.

17. Mérawa, M.; Dargelos, A.; J. Mol. Struct. 2000, 528, 37.

18. Pecul, M.; Rizzo, A.; J. Chem. Phys. 2002, 116, 1259.

19. Pecul, M.; Coriani, S.; Chem. Phys. Lett. 2002, 355, 327.

20. Miranda, S. G.; Vazquez, P. A. M.; J. Braz. Chem. Soc. 2002, 13, 1.

21. Caillie, C. V.; Amos, R. D.; Chem. Phys. Lett. 1998, 291, 71.

22. Dalskov, E. K.; Sauer, S. P. A.; J. Phys. Chem. A 1998, 102, 5269.

23. Placzek, G. Em The Rayleigh and Raman Scattering; United States Atomic Energy Commision: Lawrence Radiation Laboratory, University of California, Livermore, California, 1959, UCRL Translation $\mathrm{n}^{\circ} 526$ (L), Physics.

24. Long, D. A. Em The Raman Effect, A Unified Treatment of the Theory of Raman Scattering by Molecules; John Wiley \& Sons, LTD: England, 2002.

25. Hamming, R. W. Em Numerical Methods for Scientists and Engineers; Dover Publications, Inc.: New York, 1986.

26. Hill, T. L. Em An Introduction to Statistical Thermodynamics; Dover Publications, Inc.: New York, 1986.

27. McQuarrie, D. A. Em Statistical Mechanics; Harper \& Row: New York, 1976.

28. Wilson Jr., E. B.: Decius, J. C.: Cross, P. C. Em Molecular Vibrations, The Theory of Infrared and Raman Vibrational Spectra; Dover Publications, Inc.: New York, 1980.

29. Helgaker, T.; Jensen, H. J. A.; Joergensen, P.; Olsen, J.; Ruud, K.; Aagren, H.; Auer, A.; Bak, K.; Bakken, V.; Christiansen, O.; Coriani, S.; Dahle, P.; Dalskov, E. K.; Enevoldsen, T.; Fernandez, B.; Haettig, C.; Hald, K.; Halkier, A.; Heiberg, H.; Hettema, H.; Jonsson, D.; Kirpekar, S.; Kobayashi, R.; Koch, H.; Mikkelsen, K. V.; Norman, P.; Packer, M. J.; Pedersen, T. B.; Ruden, T. A.; Sanchez, A.; Saue, T.; Sauer, S. P. A.; Schimmelpfennig, B.; Sylvester-Hvid, K. O.; Taylor, P. R.; Vahtras, O.; Dalton release 1.2.1, an electronic structure program; University of Oslo: Norway, 2001.

30. Myers, A. B.; J. Raman Spectrosc. 1997, 28, 389.

31. Shimanouchi, T.; "Molecular Vibrational Frequencies" in NIST Chemistry WebBook; NIST Standard Reference Database 69; National Institute of Standards and Technology: Gaithersburg MD, 2001, 20899 (http:// webbook.nist.gov). 